\title{
Exploration of factors affecting prognosis of stroke preceded by transient ischemic attack
}

\author{
Nermien Naim Adly ${ }^{1}$, Safaa Hussein Ali ${ }^{2}$ \\ ${ }^{1}$ Associate Professor, Department of Geriatrics and Gerontology, Faculty of Medicine, Ain Shams University, \\ 'Lecturer, Department of Geriatrics and Gerontology, Faculty of Medicine, Ain Shams University, Cairo, Egypt
}

Background: Age is the most important risk factor for stroke. The regional brain affection was not considered in a comparison between different brain locations as a predictor for outcome in those with and without prior TIA. Aims and Objectives: Can transient Ischemic attack predict stroke prognosis? Materials and Methods: Fifty nine patients, aged $\geq 55$ years old, with recent ischemic stroke were studied, with (cases, $40.68 \%$ ) and without (controls) antecedent TIA. National Institutes of Health Stroke Scale (NIHSS), modified Rankin scale (MRS), and California risk score (CRS) were assessed. A brain computed tomography was done. Results: Older age was a predictor of more severe stroke $(P<0.001)$. Different latencies

\section{Access this article online}

Website:

http://nepjol.info/index.php/AJMS DOI: 10.3126/ajms.v7i4.14704

E-ISSN: 2091-0576 P-ISSN: 2467-9100 for TIA were studied. With reference to our controls, better prognosis was found only in cases with TIA $\leq$ seven days as a predictor for better NIHSS and MRS. In cases, CRS was a significant predictor of shorter latency. Infratentorial ischemic stroke, not supratentorial ischemic stroke, with prior TIA, was a significant predictor of lower NIHSS. Conclusion: TIA $\leq$ seven days is a predictor for better NIHSS and MRS.

Key words: Infratentorial, Latency, Prognosis, Stroke, Transient ischemic attack

\section{INTRODUCTION}

Age is an important risk factor for stroke. Stoke rates doubles for every 10 years after 55 years old. ${ }^{1}$

Ischemic preconditioning (IPC) is defined as brief periods of ischemia which are able to minimize the deleterious effects of a subsequent longer-lasting ischemic attack..$^{2}$ Moreover, the neuro-protective role of cerebral IPC is demonstrated through mammalian in vivo and in vitro studies. ${ }^{3,4}$ Focal or global cerebral ischemia induces ischemic tolerance (IT) against ischemic injury in rodents. ${ }^{3}$ In a 2 week protocol of repetitive hypoxic preconditioning, long-term protection was induced against brain injury in a mouse model of focal ischemic stroke. ${ }^{4}$ Later, other studies have demonstrated the possible protective role of transient ischemic attack (TIA) as equivalent to cerebral IPC in patients with stroke. ${ }^{5-8}$

However, not all studies have shown associations between TIAs and more favorable outcomes after stroke. In a cohort study in northern California, no association was observed between the occurrence of TIA and diminished disability due to a subsequent stroke. ${ }^{9}$ The reasons for these discrepancies remain undefined. A number of factors in clinical settings, such as the timing of TIAs relative to the occurrence of stroke (latency), cannot be controlled for as it is the case in animal studies, ${ }^{10}$ although different latency intervals were studied by different authors. ${ }^{7,11}$

Furthermore, Payabvash and his colleagues approved different ischemic vulnerabilities of the brain regions to hypoperfusion in patients with acute stroke. ${ }^{12}$ Similarly, others found different outcomes for stroke among those with supratentorial ischemic stroke (STIS) versus infratentorial ischemic stroke (ITIS) ${ }^{13}$

To our knowledge, although, certain brain regions were studied in animals ${ }^{3}$ and in humans, as Moncayo and his colleagues who studied anterior circulation strokes, ${ }^{6}$ the regional brain affection was not considered in a comparison between different brain locations as a predictor of outcome in those with and without prior TIA. 
IPC is emerging as an innovative and novel cytoprotective strategy to counter ischemic vascular disease. ${ }^{14}$ Since stroke and TIA mostly affect the aging population, it is important to decide whether TIA remains effective protection in aged patients with underlying vascular disease. $^{7}$

Many tools such as California risk score (CRS), which has the best specificity among tools that can be done by emergency room physician as ABCD (concerned with age, blood pressure, clinical feature and duration) and $\mathrm{ABCD}_{2}$, (concerned with age, blood pressure, clinical feature, duration and diabetes). ${ }^{15}$ CRS was developed to predict risk of stroke. ${ }^{16}$ However, could this tool be useful in predicting earlier stroke? And is it related to subsequent stroke severity?

Therefore, the aims of this study were to determine whether prior TIA could affect the outcome of the subsequent acute ischemic stroke in patients aged $\geq 55$ years old, does latency or regional affection of the brain have a role? And could CRS be related to a subsequent stroke outcome or latency?

\section{MATERIALS AND METHODS}

Case-control study was conducted. Patients with acute ischemic stroke admitted to Geriatric department and internal medicine departments, over a period of six months, aged $\geq 55$ years, with (cases) and without (controls) antecedent TIA, were studied.

Informed oral or written consent was obtained and patient anonymity was preserved. The study was approved by the ethical committee of our department.

Patients were excluded if they had intracranial hemorrhage, previous strokes or TIAs with lesion on cerebral computed tomography (CT), pre-existing dementia, or lacunar strokes.

Comorbidities, including chronic heart failure, chronic obstructive pulmonary disease, coronary heart disease, atrial fibrillation, diabetes and hypertension, were defined by patient history and clinical examination. The stroke severity was assessed by the National Institutes of Health Stroke Scale (NIHSS), ${ }^{17}$ and disability was assessed with the modified Rankin scale (MRS), ${ }^{18}$ within 24 hours after admission.

\section{Radiological data}

A brain CT scan was performed at admission, and sometimes was repeated after 48 hours, or 72 hours from the acute stroke onset.

\section{Laboratory data}

During the medical evaluation, blood sample was drawn for all participants after fasting for 12 hours for cholesterol, triglycerides, high density lipoprotein (HDL) and low density lipoprotein (LDL). These were measured using standard laboratory methods.

\section{Data processing and statistical analysis}

The statistical analysis was performed using the $16^{\text {th }}$ version of SPSS. Qualitative data were presented in the form of frequency tables. Quantitative data were presented in the form of means \pm SD (for parametric data) or median and interquartile values (for non-parametric data).

Normality distribution was tested using one sample Kolmogorov Smirnov test. Regarding quantitative data, differences between groups were assessed using the Student's t test, or ANOVA, LSD was used in post hoc test, for parametric data and Mann Whitney U or Kruskal-Wallis test for non-parametric data. Regarding qualitative data, the Chi-square test or Fisher's Exact test was used to compare between groups.

Generalized linear method (GLM) was used to detect the significant predictors of outcome(as assessed by NIHSS and MRS) among those with no previous TIA and those with previous TIA (different models were used to compare different latencies with the following cut offs seven, 14, 21 and 30 days, each latency was studied separately), with further adjustment for age $\geq 75$ years old in additional models, as a retrospective clinical study suspected that IT may not be occurring in the elderly, aged around 75 years old. ${ }^{19}$

CRS and older age group $\geq 75$ years old were tested as predictors of stroke outcome.

Receiver operator curve (ROC) was used to evaluate the discriminatory performances of the significant predictors of outcome, derived from GLM, for the occurrence of better outcome in the subsequent stroke.

\section{RESULTS}

Fifty nine patients with recent stroke were studied, $40.68 \%$ had antecedent TIA. Among cases, $20.8 \%$ had TIA within seven days, 37.5\% had TIA within 14 days, 50\% had TIA within 21 days and $62.5 \%$ had TIA within 30 days. Median of latency and inter-quartiles were 25.5 (8.5-57) days. Only one case had two TIAs, the others had one TIA. The duration of TIA was more than 10 minutes in the study group.

There was no significant difference between cases and controls regarding age, gender, smoking status, 
comorbidities, or stroke severity tools (NIHSS and MRS) (Table 1).

By studying significant predictors of NIHSS, using GLM, CRS was not a significant predictor of a subsequent stroke severity $(\mathrm{P}=0.23)$, in cases (Table 2$)$.

Among cases and controls, older age ( $\geq 75$ years old) was a significant predictor of a subsequent stroke severity ( $\mathrm{P}$ $<0.001)$ as assessed by NIHSS and MRS. In addition, controls (those with stroke without antecedent TIA) were entered in regression with cases (those with stroke with antecedent TIA) with classification of cases using different latencies with the following cut offs seven, 14,
21 and 30 days in different models. With reference to our controls, significant result was obtained only in cases with TIA $\leq$ seven days as a predictor for better NIHSS $(\mathrm{P}<0.001)$ rather than those with latency $>$ seven days, other latencies gave no significant results. This significance was maintained after adjustment for older age group (Table 2).

By studying regional brain affection as a predictor for NIHSS, controls were studied versus cases with STIS and cases with ITIS, in GLM. With reference to our controls, ITIS with prior TIA was the only significant predictor of lower NIHSS ( $\mathrm{P}<0.001)$ (Table 2), HDL was adjusted for, as it was the only significant different variable between

\section{Table 1: Descriptive statistics for cases and controls}

\begin{tabular}{|c|c|c|c|}
\hline Variables & Cases $(n=24)$ & Controls $(n=35)$ & $P$ value \\
\hline Age, mean $\pm S D$ (years) & $65.37 \pm 9.2$ & $68.14 \pm 10.4$ & 0.29 \\
\hline Male gender, $\mathrm{n}(\%)$ & $12(50 \%)$ & $16(45.7 \%)$ & 0.75 \\
\hline Current smokers, n (\%) & $7(29.2 \%)$ & $6(17.1 \%)$ & 0.22 \\
\hline Ex_smokers, $\mathrm{N}(\%)$ & $0(0 \%)$ & $3(8.6 \%)$ & \\
\hline \multicolumn{4}{|l|}{ Comorbidities, n (\%) } \\
\hline Heart failure & $1(4.2 \%)$ & $2(5.7 \%)$ & $1^{\dagger}$ \\
\hline IHD & $4(16.7 \%)$ & $7(20 \%)$ & $1^{\dagger}$ \\
\hline Atrial fibrillation & $2(8.3 \%)$ & $2(5.7 \%)$ & $1 \dagger$ \\
\hline COPD & $1(4.2 \%)$ & $5(14.3 \%)$ & 0.39 \\
\hline Diabetes mellitus & $15(62.5 \%)$ & $18(51.4 \%)$ & 0.4 \\
\hline Hypertension & $17(70.8 \%)$ & $20(57.1 \%)$ & 0.29 \\
\hline \multicolumn{4}{|l|}{ Lipid profile, mean $\pm S D$} \\
\hline Total cholesterol mg/dl & $155.35 \pm 67.7$ & $153.15 \pm 71.6$ & 0.92 \\
\hline LDL mg/dl & $103.5 \pm 49.7$ & $86.33 \pm 44.2$ & 0.30 \\
\hline $\mathrm{HDL} \mathrm{mg/dl}$ & $29.56 \pm 17.3$ & $18.5 \pm 10.97$ & $0.04^{*}$ \\
\hline Triglycerides mg/dl & $127.26 \pm 78.9$ & $112.34 \pm 57.9$ & 0.49 \\
\hline \multicolumn{4}{|c|}{ Outcome scores, median (interquartile) } \\
\hline NIHSS & $8(4-19)$ & $11(5-18)$ & 0.79 \\
\hline Modified Rankin scale & $5(4-5)$ & $5(4-5)$ & 0.95 \\
\hline \multicolumn{4}{|c|}{ Distribution of computed tomography findings } \\
\hline Supratentorial lesion & $18(75 \%)$ & $21(60 \%)$ & 0.5 \\
\hline Infratentorial lesion & $4(16.7 \%)$ & $7(20 \%)$ & \\
\hline Both & $0(0 \%)$ & $2(5.7 \%)$ & \\
\hline No lesion specified & $2(8.3 \%)$ & $5(14.3)$ & \\
\hline
\end{tabular}

*Significant $\mathrm{P}$ value. ${ }^{\dagger}$ Fisher Exact test was used, *Significant, Values were expressed in form of mean \pm SD or median (interquartile values) for quantitative data and number (\%) for qualitative data, COPD: Chronic obstructive lung disease, HDL: High density lipoprotein, IHD: Ischemic heart disease, LDL: Low density lipoprotein, NIHSS: National Institutes of health stroke scale

Table 2: Generalized linear method analysis of NIHSS predictors

\begin{tabular}{lccccc}
\hline Predictors & $\begin{array}{c}\text { Beta } \\
\text { coefficient }\end{array}$ & O.R. & $\begin{array}{c}\mathbf{9 5 \%} \text { CI } \\
\text { (lower-upper) of beta }\end{array}$ & $\begin{array}{c}\mathbf{9 5 \%} \text { Cl } \\
\text { (lower-upper) of O.R. }\end{array}$ & $\begin{array}{c}\text { P value } \\
(1.8-5.9)\end{array}$ \\
\hline $\begin{array}{l}\text { Age } \geq 75 \text { years old } \\
\text { TIA } \leq 7 \text { days without adjustment for age } \\
\quad \text { cases with TIA } \leq 7 \text { days }\end{array}$ & 1.18 & 3.25 & $(-1.78--0.57)$ & $<0.001^{*}$ \\
$\begin{array}{l}\text { TIA } \leq \text { seven days, with adjustment for age } \\
\quad \text { cases with TIA } \leq \text { seven days }\end{array}$ & -8.34 & $2.4^{\dagger}$ & $(-9.563--7.11)$ & $\left(0.75^{\dagger}-0.6\right)$ & $<0.001^{*}$ \\
$\begin{array}{l}\text { California risk score (among cases) } \\
\quad \text { California risk score }\end{array}$ & -8.189 & $2.8^{\dagger}$ & $(-9.430--6.95)$ & $\left(0.8^{\dagger}-0.0008\right)$ & $<0.001^{*}$ \\
$\begin{array}{l}\text { STIS and ITIS with prior TIA, with adjustment for HDL } \\
\text { ITIS }\end{array}$ & -0.3 & 0.74 & $(-0.83--0.23)$ & $(0.4-0.8)$ & 0.23 \\
\hline
\end{tabular}

*Significant P value, 'Data multiplied by $10^{-4}$, Cl: confidence interval, ITIS: infratentorial ischemic stroke, NIHSS: national Institutes of health stroke scale, OR: odd ratio, STIS: supratentorial ischemic stroke, TIA: transient ischemic attack 
groups (it was significantly higher in those with STIS and prior TIA than ITIS with prior TIA, $\mathrm{P}=0.024)$.

Among cases, using GLM, CRS was not a significant predictor of MRS $(\mathrm{P}=0.34)$ (Table 3$)$.

Among cases and controls, older age was not a significant predictor of the subsequent stroke disability $(\mathrm{P}=0.9)$. In addition, controls were entered in regression with cases with classification of cases using the previous latencies in different models. With reference to our controls, significant result was obtained only in cases with TIsA $\leq$ seven days as a predictor of better MRS $(\mathrm{P}=0.002)$ rather than those with latency $>$ seven days. This significance was maintained after adjustment for older age group (Table 3).

In cases, $C R S$ was a significant predictor of shorter latency $(\beta=-8.1,95 \%$ C.I. $=1.8-4.6, \mathrm{OR}=2.9, \mathrm{P}<0.001)$.

There was no significant difference between controls, cases with TIA $\leq$ seven days or cases with TIA $>$ seven days in baseline characteristics or regional brain affection by CT.

By using ROC, patients with TIA $\leq$ seven days, had moderate accuracy for identification of patients with better NIHSS with AUC $=0.779,95 \%$ C.I. $=(0.63-0.92)$, at cut off $\leq 14$, sensitivity $=41 \%$ and specificity $=100 \%$; and at cut off $\leq 7$, sensitivity $=63 \%$, and specificity $=100 \%$.

By using ROC, patients with TIA $\leq$ seven days, had moderate accuracy for identification of patients with better MRS with AUC $=0.69,95 \%$ C.I. $=(0.39-0.98)$, at cut off $\leq 3$, sensitivity $=89 \%$ and specificity $=60 \%$.

\section{DISCUSSION}

IPC is emerging as an innovative and novel cytoprotective strategy to counter ischemic vascular disease. ${ }^{14}$

Therefore, we studied the role of previous TIA in IPC, and with reference to our controls, better prognosis was found only in cases with TIA $\leq$ seven days as a predictor for better NIHSS and MRS.

The current results were not similar to Johnston's results, who studied a comparison between different latencies: less than one day, one- seven days, and up to 90 days in separate categories, as preconditioning was not expected in the latter group. The latency was similar in those with disabling and non-disabling stroke. The stroke was disabling in $85 \%$ when it occurred in $\leq$ seven days of the TIA and in $84 \%$ of those with stroke occurring $>$ seven days $(\mathrm{P}=0.83) .{ }^{9}$.

The disagreement with the current results might be related to the different criteria of the patients, as we excluded lacunar or previous stokes, while his study didn't explore any exclusion criteria. In the current study, patients with lacunar stroke were excluded because prior TIA was not associated with a favorable outcome in them, ${ }^{20}$ as lacunar stroke arteries have a terminal type of distribution rather than the rich collateralization of cortical arteries. ${ }^{21}$

The current results were not in accordance with the retrospective study performed by Della Morte and his colleagues, who studied 42 patients with previous TIA, within 72 hours of stroke onset, versus those without previous TIA among elderly presented with non-lacunar stroke, with mean of age 75 years old. They found no significant difference in the NIHSS or MRS scores. ${ }^{11}$ There were no details about infarct location or frequency of TIAs.

Some authors performed critical stenosis in left common carotid artery in mice, $45 \%$ to $65 \%$ of baseline cortical perfusion was diminished, then they found significant preservation of cortical perfusion, with attenuation of infarct size and neurologic deficits, after middle cerebral artery (MCA) occlusion in day 14 and day 28 groups compared with day 0,4 , and sham groups. ${ }^{22}$ They concluded that it may need longer time to develop collaterals into large vessels after its critical stenosis. ${ }^{23}$ This might partially explain the difference with our latency, as basal ganglion involvement was present in only 3 of our cases,

\begin{tabular}{|c|c|c|c|c|c|}
\hline Predictors & $\begin{array}{c}\text { Beta } \\
\text { coefficient }\end{array}$ & O.R. & $\begin{array}{c}95 \% \mathrm{Cl} \\
\text { (lower-upper) of beta }\end{array}$ & $\begin{array}{c}95 \% \mathrm{Cl} \\
\text { (lower-upper) }\end{array}$ & $P$ value \\
\hline Age $\geq 75$ years old & 0.03 & 1.03 & $(-0.53-0.59)$ & $(0.59-1.8)$ & 0.9 \\
\hline $\begin{array}{l}\text { TIA } \leq \text { seven days without adjustment for age } \\
\text { cases with TIA } \leq \text { seven days }\end{array}$ & -1.56 & 0.21 & $(-2.55--0.58)$ & $(0.1-0.6)$ & $0.002^{*}$ \\
\hline $\begin{array}{l}\text { TIA } \leq \text { seven days, with adjustment for age } \\
\text { cases with TIA } \leq \text { seven days }\end{array}$ & -1.48 & 0.23 & $(-2.43--0.52)$ & $(0.1-0.5)$ & $0.002^{*}$ \\
\hline California risk score (among cases) & & & & & \\
\hline California risk score & -0.23 & 0.79 & $(-0.71-0.24)$ & $(0.5-1.3)$ & 0.34 \\
\hline
\end{tabular}


as occlusion of lenticulostriate arteries is an early sign of MCA occlusion. ${ }^{24}$

Other authors studied IT upon rats with several reperfusion periods (ie, two, six, and 12 hours and one, two, seven, 14, and 21 days) between IPC and final ischemia and found that IPC induces IT that is dependent on new protein(s) synthesis that occurs at the IPC brain site one to seven days after IPC which contributes to the neuroprotection. In this model temporary MCA occlusion for ten minutes was used for IPC before permanent MCA occlusion ( $\mathrm{n}=$ seven to nine per group) to establish IT compared with sham group $(n=22) .{ }^{25}$

A recent experimental TIA approved role of IPC in rats, when stroke was induced 2 weeks later ${ }^{4}$, however this prolonged IT could be related to the repeated hypoxic exposure in the IPC phase, as repeated TIA per se could have a role in prolongation of IPC. ${ }^{26}$

Furthermore, our results were in accordance with Schaller, who studied 11 cases versus 119 controls and found that TIA that occurred within one-seven days may lead to IT against a subsequent ischemic stroke as demonstrated by the significant diminished infarct extension throughout neuroradiological studies. ${ }^{7}$

Weih and his colleagues studied 37 cases versus 111 controls and found that TIA before stroke was associated with less severe stroke, as assessed by Canadian Neurological Scale, on admission and with follow-up, The median of latency was 21 days (range 6 hours to 2 years), $6 \%$ of their cases had small vessel disease. There was no significant difference in baseline characteristics. ${ }^{5}$

The wide variation between studies, regarding the protective latency in different studies, precludes the possible role for other underlying factors. Indeed, not all authors had same exclusion criteria or gave comment about frequency of TIA in their patients, its duration or regional affection of the brain that might give a better comparison between studies.

As we found that, with reference to our controls, ITIS with prior TIA, rather than STIS with prior TIA, was a significant predictor for better NIHSS. This agrees with De Haan and his colleagues who reported a better quality of life in ITIS than in STIS patients. ${ }^{13}$

This could point to one of other factors that might affect stroke outcome after TIA. This finding might be explained by molecular studies for IT in the brain.

The sodium calcium exchanger (NCX) has three isoforms: NCX1, NCX2, and NCX3 which regulate Ca2+ homeostasis. The two isoforms NCX1 and NCX3 can be involved in IT. ${ }^{27}$

NCX1 was found to mainly control the input of the cerebellum, and NCX2 was seen more extensively distributed in supratentorial areas rather than infratentorial areas, as Canitano and his colleagues presented its distribution in each of the brain areas in the rat central nervous system. ${ }^{28}$

In the current study, patients presented with stroke that had antecedent TIA within seven days were a significant predictor of favorable outcome, lower NIHSS and MRS, even after adjustment for the older age group.

Older age was a significant predictor of stroke severity at admission, which is in accordance with Corso and his colleagues who showed that very old age is an independent predictor of stroke severity at admission in a population based study, among those with first ever ischemic-stroke. ${ }^{29}$

However, older age was not a significant predictor of initial stroke disability. This is in accordance with Bagg and his colleagues who showed that age has an insignificant clinical impact, as a small variation in functional outcome (about $1 \%$ ) can be attributed solely to age. ${ }^{30}$

CRS was not a significant predictor for NIHSS or MRS. However, its increase was a significant predictor of earlier stroke following TIA. This later finding was in consistence with a study by Johnston and his colleagues upon $\mathrm{ABCD}_{2}$ score, in which higher score was associated with higher percentage of stroke within 2 days. ${ }^{31}$

Although CRS could be suspected to influence stroke severity due its sub items as age. However, it was not related to severity as its higher score constituted a higher risk for earlier stroke that might benefit from preconditioning.

\section{Limitations}

The small sample size was a limitation of the current work. However this is attributed to the limited number of patients at this age group who presented with first stroke plus previous TIA, and this limitation was found in other studies, as Weih and his colleagues studied 37 cases, and Wegener and his colleagues studied 16 cases. $^{5,8}$ Our TIA cases were $40.68 \%$ while in Weih's study, for example, cases were $25 \%{ }^{5}$

\section{Recommendations}

We could suggest considering latency with its cut off of seven days in further tools which predict stroke severity. Further elucidation of the role of CRS in prediction of latency need to be clarified in future researches. We would 
like to suggest the consideration of seven days as a possible protective latency in future experimental TIA researches combined with the observation of regional brain affection.

\section{Competing interests}

No conflict of interest has been declared by the authors.

\section{ACKNOWLEDGMENTS}

The research team acknowledges the patients and their families.

\section{Disclosure statement}

No potential conflicts of interest were disclosed.

\section{REFERENCES}

1. Control C. Stroke facts and statistics. In: centers for disease control (Ed). Heart disease and stroke prevention. 2006. Atlanta, Ga: Department of Health and Human Services.

2. Murry C, Sennings R and Reimer K. Preconditioning with ischemia: A delay of lethal cell injury in ischemic myocardium. Circulation 1986; 4: 1124-1136.

3. Dave K, Saul I, Busto R, Ginsberg M, Sick T and Pérez-Pinzón M. Ischemic preconditioning preserves mitochondrial function after global cerebral ischemia in rat hippocampus. J Cereb Blood Flow Metab 2001; 21: 1401-1410.

4. Poinsatte K, Selvaraj U, Ortega S, Plautz E, Kong X, Gidday J, et al. Quantification of $n$ eurovascular protection following repetitive hypoxic preconditioning and transient middle cerebral artery occlusion in mice. J Vis Exp 2015; 4 (99): e52675.

5. Weih M, Kallenberg K, Bergk A, Dirnagl U, Harms L, Wernecke K, et al. Attenuated stroke severity after prodromal TIA: A role for ischemic tolerance in the brain? Stroke 1999; 30: 1851-1854.

6. Moncayo J, de Freitas G, Bogousslavsky J, Altieri M and van Melle G. Do transient ischemic attacks have a neuroprotective effect? Neurology2000; 54: 2089-2094.

7. Schaller B. Ischemic preconditioning as induction of ischemic tolerance after transient ischemic attacks in human brain: Its clinical relevance. Neurosci Lett 2005; 377: 206-211.

8. Wegener S, Gottschalk B, Jovanovic V, Knab R, Fiebach J and Schellinger P. MRI in Acute Stroke Study Group of the German Competence Network Stroke. Transient ischemic attacks before ischemic stroke: Preconditioning the human brain? A multicenter magnetic resonance imaging study. Stroke 2004; 35: 616-621.

9. Johnston S. Ischemic preconditioning from transient ischemic attacks? Data from the Northern California TIA study. Stroke 2004; 35: 2680-2682

10. Sandu N, Cornelius J, Filis A, Arasho B, Perez-Pinzon M and Schaller B. Ischemic tolerance in stroke treatment. Expert Rev Cardiovasc Ther 2009; 7: 1255-1261.

11. Della Morte D, Abete P, Gallucci F, Scaglione A, D'Ambrosio D, Gargiulo G, et al. Transient ischemic attack before nonlacunar ischemic stroke in the elderly. J Stroke Cerebrovasc Dis 2008; 17(5): 257-62.

12. Payabvash S, Souza L, Wang Y, Schaefer P, Furie K, Halpern E, et al. Regional ischemic vulnerability of the brain to hypoperfusion: the need for location specific computed tomography perfusion thresholds in acute stroke patients. Stroke 2011; 42(5): 1255-1260.

13. De Haan R, Limburg M, Van der Meulen J, Jacobs $H$ and Aoronson N. Quality of life after strokes: impact of stroke type and lesion location. Stroke 1995; 26: 402-428.

14. Koch S, Della-Morte D, Dave K, Sacco R and PerezPinzon M. Biomarkers for ischemic preconditioning: finding the responders. Journal of Cerebral Blood Flow \& Metabolism 2014; 34: 933-941.

15. Gupta H, Farrell A and Mittal M. Transient ischemic attacks: predictability of future ischemic stroke or transient ischemic attack events. Ther Clin Risk Manag 2014; 10: 27-35.

16. Johnston S, Gress D, Browner W and Sidney S. Short-term prognosis after emergency department diagnosis of TIA. JAMA 2000; 284(22): 2901-2906.

17. Lyden $\mathrm{P}$, Brott $\mathrm{T}$, Tilley B, Welch $\mathrm{K}$, Mascha E, Levine S, et al. Improved reliability of the NIH stroke scale using video training: NINDS TPA stroke study group. Stroke 1994; 25: 2220-2226.

18. Van Swieten J, Koudstaal $P$, Visser M, Schouten $H$ and van Gijn J. Interobserver agreement for the assessment of handicap in stroke patients. Stroke 1988; 19: 604-607.

19. Tomida S, Nowak T, Vass K, Lohr J and Klatzo I. Experimental model for repetitive ischemic attacks in the gerbil: the cumulative effect of repeated ischemic insults. J Cereb Blood Flow Metab 1987; 7: 773-782.

20. Arboix A, Cabeza N, García-Eroles L, Massons J, Oliveres M, Targa C, et al. Relevance of transient ischemic attack to early neurological recovery after nonlacunar ischemic stroke. Cerebrovasc Dis 2004: 18:304-311.

21. Del B, Palumbo V, Lamassa M, Saia V, Piccardi B and Inzitari D. Progressive lacunar stroke: review of mechanisms, prognostic features, and putative treatments. Int J Stroke 2012; 7: 321-329.

22. Kitagawa K, Yagita Y, Sasaki T, Sugiura S, Omura-Matsuoka E, Mabuchi T, et al. Chronic mild reduction of cerebral perfusion pressure induces ischemic tolerance in focal cerebral ischemia. Stroke 2005; 36(10): 2270-2274.

23. Buschmann $I$ and Schaper $W$. The pathophysiology of the collateral circulation (arteriogenesis). J Pathol 2000; 190: 338-342.

24. Nakano $\mathrm{S}$, Iseda $\mathrm{T}$, Kawano $\mathrm{H}$, Yoneyama $\mathrm{T}$, Ikeda $\mathrm{T}$ and Wakisaka S. Correlation of Early CT Signs in the Deep Middle Cerebral Artery Territories with Angiographically Confirmed Site of Arterial Occlusion. Am J Neuroradiol 2001; 22: 654-659.

25. Barone F, White R, Spera P, Ellison J, Currie R, Wang X, et al. Ischemic preconditioning and brain tolerance: temporal histological and functional outcomes, protein synthesis requirement, and interleukin-1receptor antagonist and early gene expression. Stroke 1998; 29: 1937-1950.

26. Lin X, Zhao L, Wang X, Zhu J and Tian X. The Clinical Study of Transient Ischemic Attack on Cerebral Ischemic Tolerance. Asian Case Reports in Vascular Medicine 2012; 1: 1-4.

27. Pignataro G, Boscia F, Esposito E, Sirabella R, Cuomo O, Vinciguerra A, et al. NCX1 and NCX3: two new effectors of delayed preconditioning in brain ischemia. Neurobiol Dis 2012; 45: 616-623.

28. Canitano A, Papa M, Boscia F, Castaldo P, Sellitti S, Taglialatela $M$, et al. Brain distribution of the $\mathrm{Na}+/ \mathrm{Ca} 2+$ exchanger-encoding genes NCX1, NCX2, and NCX3 and their related proteins in the central nervous system. Ann N Y Acad Sci 2002; 976: 394-404.

29. Corso G, Bottacchi E, Tosi P, Caligiana L, Lia C, Morosini M, et al. Outcome Predictors in First-Ever Ischemic Stroke Patients: A Population-Based Study. International Scholarly Research 
Notices 2014; Article ID 904647. Retrieved from http://dx.doi. org/10.1155/2014/904647

30. Bagg S, Pombo A and Hopman W. Effect of age on functional outcomes after stroke rehabilitation. Stroke 2002; 33(1): 179-185.
31. Johnston S, Rothwell P, Huynh-Huynh M, Giles M, Elkins J and Sidney S. Validation and refinement of scores to predict very early stroke risk after transient ischemic attack. Lancet 2007; 369: 283-292.

\section{Authors Contribution:}

NAA - Concept and design of the study, reviewed the literature, manuscript preparation and statistically analyzed and interpreted the data; SHA - Concept,

collected data and critical revision of the manuscript. Both authors reviewed the final script.

Source of Support: Nil, Conflict of Interest: None declared. 